\title{
La autonomía universitaria que fortalece la gestión curricular intercultural
}

Letisia Castillo Gómez ${ }^{1}$

\section{Resumen}

En este artículo se intentó armonizar a través de la revisión bibliográfica, dos grandes campos en el mundo de la Educación Superior: la autonomía universitaria y la gestión curricular. Presenta las dimensiones de la autonomía institucional y académica, así como la gestión curricular como parte esencial del cumplimiento del modelo educativo con identidad. Asimismo, refleja la necesidad de un verdadero ejercicio autonómico para poder llevar a cabo todas las acciones educativas que respondan de manera pertinente a los sueños, aspiraciones y demandas educativas de los pueblos con un carácter intercultural. Por último, se resalta la existencia de instituciones de Educación Superior con perfiles particulares que valoran la diversidad humana y natural como la principal riqueza.

Palabras clave: Autonomía universitaria; gestión curricular; gestión curricular intercultural.

\section{Summary}

This article attempts to harmonize through the bibliographical revision, two large fields in the higher education world: university autonomy and curriculum management. It shows the dimensions of institutional and academic autonomy, as well as the curricular management as an essential part of the fulfillment of an educational model with identity. It also reflects the need for a true autonomic exercise to be able to carry out all educational actions that respond pertinently to the dreams, aspirations and educational demands of the people with an intercultural approach. Finally, it highlights the existence of Higher Education institutions with particular profiles that value human and natural diversity as the main wealth.

Keywords: University autonomy; curriculum management; intercultural curriculum management.

1 Máster en Docencia Universitaria, Directora Académica General de la URACCAN, letycuba2oo1@yahoo.com, academica.general@ uraccan.edu.ni 


\section{Introducción}

La historia define que la Universidad es una institución social, cultural y científica por excelencia, fruto de escenarios muy diferentes a los actuales, pero con la vigencia que requiere la era del conocimiento. Ella atesora desde sus años iniciales el ejercicio de la libertad, concretado en su Autonomía. Como principio se puntualiza en autonomía orgánica, académica, administrativa y financiera de la institución respecto a factores externos. Es así que esta libertad se traduce en la independencia política y administrativa de la Universidad, para el debate, análisis y búsqueda de la verdad, lo que se debe expresar en todo el quehacer, incluyendo la gestión del currículo.

Sin duda alguna, la Autonomía Universitaria es un invalorable instrumento que tenemos a nuestra disposición las Universidades Latinoamericanas. Pero ¿será la gestión curricular intercultural, dentro del marco de autonomía, uno de los elementos catalizadores para poner en marcha modelos educativos innovadores e integrales, que abarquen contenidos con enfoque de equidad, identidad e interculturalidad, y coloquen en relieve las epistemologías y las necesidades de formación de los diferentes pueblos originarios? Además ésta ¿garantiza la libertad, dentro de la legalidad, para cuestionar y poner a prueba los conocimientos recibidos, proponer nuevas ideas y sostener opiniones polémicas?

En este trabajo interesa conjugar la autonomía universitaria con la responsabilidad de una gestión curricular intercultural para poder problematizar la manera de cómo la universidad debe responder pertinentemente a las necesidades, sueños, demandas, aspiraciones y proyectos de los diferentes pueblos, considerando al ser humano como una "hebra importante del tejido vivo" y sujeto de derechos individuales y colectivos.

Se aborda el debate en dos planos. El primero referido a comprender los conceptos de los temas principales. El segundo plano a destacar la naturaleza histórica, política y cultural de las Universidades Indígenas, Interculturales y Comunitarias, que garantiza una gestión curricular intercultural y fortalece la autonomía universitaria, como una reivindicación de los pueblos a ejercer sus derechos colectivos, revitalizar, desarrollar y transmitir a las generaciones futuras sus historias, idiomas, tradiciones, saberes, prácticas y valores comunitarios.

\section{Materiales y método}

El presente trabajo es un artículo de revisión bibliográfica. Para el desarrollo del mismo, se realizó una consideración descriptiva de la bibliografía publicada del tema, para lo cual se utilizaron varias fuentes documentales. Para la búsqueda y selección de la información se tomaron criterios como: pertinencia con el contexto, relevancia sobre el tema y coherencia con las preguntas centrales. Seguidamente se situó en la perspectiva deseada y se elaboró el documento final. 


\section{Desarrollo}

\section{La Autonomía institucional y académica: aproximaciones conceptuales}

El bien de la autonomía universitaria se enmarca particularmente en la solicitud de movimientos universitarios en Latinoamérica, de la "Reforma Universitaria", la que se propuso dos conquistas claves: la autonomía y el cogobierno universitario, lo que no incluyó la estructura académica. Como bien apunta Tünnermann (1997), la Reforma, en cuanto a la organización de la Universidad, se mantuvo en el área de la ordenación de la misma como institución autónoma y democrática. No dirigió sus dardos, con igual empeño, en contra de la estructura académica, aunque se preocupó de los aspectos relacionados con la docencia y los métodos de enseñanza (p.74).

En 1918 los estudiantes de la Universidad Nacional de Córdoba, en Argentina, iniciaron una huelga universitaria en reclamo de profundas reformas universitarias que se convirtieron, rápidamente, en un amplio movimiento en América Latina, con impacto en otras regiones. En Nicaragua se materializó cuarenta años después, específicamente el 25 de marzo de 1958, cuando el presidente de la República Sr. Luis Somoza, decretó la ley de Autonomía Universitaria en respuesta a las perseverantes gestiones del Dr. Mariano Fiallos Gil, "Padre de la Autonomía Universitaria" en nuestro país.

En este contexto, es preciso resaltar el concepto de Autonomía. El más divulgado y generalmente aceptado lo ofreció en 1953 la Unión de Universidades de América Latina (UDUAL, 1954), la que establece lo siguiente:

La autonomía de la Universidad es el derecho de esta Corporación a dictar su propio régimen interno y a regular exclusivamente sobre él; es el poder de la Universidad de organizarse y de administrarse a sí misma. Dicha autonomía es consustancial a su propia existencia y no a una merced que le sea otorgada -y debe ser aseguradacomo una de las garantías constitucionales (p.99).

En la República de Nicaragua en abril de 1990, se marcó un hito importante en la historia de la educación terciaria, la Asamblea Nacional aprobó la Ley 89 "Ley de Autonomía de las Instituciones de Educación Superior", la que en su considerando II expresa:

Que la autonomía universitaria, por la que se ha luchado en Nicaragua desde hace años, implica la capacidad de la Universidad para formular su propia legislación interna, designar sus autoridades, autogobernarse y planificar su actividad académica, así como disponer de sus fondos con entera libertad.

Asimismo, en el artículo 8 se declara en qué consiste el gozo de autonomía orgánica, autonomía administrativa, autonomía financiera o económica y autonomía 
docente o académica. Logrando que la universidad sea un refugio seguro para la libertad y sosteniendo que debe ser autónoma y auto-gobernada, que debe elegir sus propias autoridades, decidir sus adecuados estatutos, políticas, reglamentos, planes y programas de estudio.

Se entiende desde la ley 89 que la Autonomía docente o académica: implica que pueden las Universidades por sí misma nombrar y remover el personal docente y académico, por medio de los procedimientos y requisitos que ellas mismas señalen; seleccionar a sus alumnos, mediante las pruebas y condiciones necesarias; elaborar y aprobar sus planes y programas de estudios y de investigación, etc.

Todo lo anterior nos ofrece un escenario fértil para poder desarrollar procesos y proyectos educativos innovadores, pertinentes con la diversidad cultural, que ponga en práctica la armonía entre los saberes universales y los saberes locales, originarios y contemporáneos y nos lancemos por fin a un verdadero diálogo de saberes con equidad epistémica y prontamente social.

Por tanto la universidad contemporánea en el goce pleno de su autonomía, debe según Tünnermann (1996):

...reconocer y actuar en consecuencia con la diversificación de las sociedades en el mundo, la composición cada vez más multicultural de éstas, las características de la masificación, las estructuras de comunicación e información, la incorporación de tecnologías en la vida cotidiana, la reducción de la distancia entre lo público y lo privado, el acceso de los ciudadanos a formas de búsqueda del conocimiento distintas a las que ella emplea, las nuevas dimensiones del trabajo basadas en la capacidad de iniciativa personal y colectiva y en la responsabilidad de las decisiones, la interdisciplinariedad de los puestos de trabajo y la movilidad permanente de los perfiles profesionales, la movilidad geográfica y cultural, la mutación sin pausa de la sociedad definida por la incertidumbre y la complejidad y la reducción del Estado-nación por superestructuras regionales, económicas y sociales (p.44).

En este sentido, Orozco (1994), en conferencia frente al papel de la universidad, señala:

...la universidad representa aquel espacio para la búsqueda del conocimiento, la libertad de pensamiento, la excelencia, la posibilidad de crítica, de diálogo dentro de un clima científico de honestidad intelectual. Esta visión de la universidad contextualizada, enfocada y sintonizada dentro del más amplio concepto de desarrollo humano, de plano se contrapone a aquella que sólo ve en ella una máquina para producir profesionales (p.5). 
Estas palabras son una invitación a reflexionar sobre el verdadero sentido de las universidades en estos momentos. Mejorar la calidad y pertinencia de la docencia, hacer una mejor gestión de ella no tiene sentido en sí misma, sino se convierte en un desafío permanente para lograr el cumplimiento de su función esencial: la formación integral de la persona y el ciudadano.

El término, formación integral, por su carácter tan general resulta necesario precisarlo mejor para entender en cada caso qué se quiere decir cuando se emplea. En síntesis, expresa la pretensión de centrar el quehacer de las universidades en la formación de valores en los profesionales de forma más plena, dotándolos de cualidades de alto significado humano, capaces de comprender la necesidad de poner sus conocimientos al servicio de la sociedad en lugar de utilizarlos sólo para su beneficio personal. Implica también la necesidad de lograr un profesional creativo, independiente, preparado para asumir su autoeducación durante toda la vida; que sea capaz de mantenerse constantemente actualizado, utilizando igualmente las oportunidades ofrecidas por las universidades de atender al profesional con una educación posgraduada que responda a las necesidades del desarrollo del país.

\section{La gestión curricular}

La universidad en su sentido amplio orienta y dirige las acciones educativas de una manera específica en cada proyecto, por lo que declara qué se debe enseñar y qué deben aprender los estudiantes. En este escenario el currículo juega un papel fundamental como proyecto articulador entre el proyecto educativo institucional y el proyecto histórico cultural de los pueblos y la sociedad, desarrollado a través de las acciones que realizan docentes y estudiantes para lograr el efecto formativo que se espera.

A como lo indica Álvarez (1998) que lo curricular, como lo propio de la institución educativa -en nuestro caso la universidad-y como el aspecto mediador entre el proyecto educativo universitario y las acciones que en la institución desarrollan profesores y alumnos a través de las diversas prácticas educativas responde de una manera u otra al proyecto histórico cultural de la sociedad donde está inserta la institución.

Con todo lo anterior es importante poner mucha atención a una efectiva gestión curricular y su importante papel. Cabe entonces definir qué entendemos por gestión curricular. Según el Centro Universitario de Desarrollo (CINDA) y el Programa de Intercambio Universitario entre la Unión Europea y América Latina (ALFA) (1996):

[...] es un término amplio que implica un conjunto de fases y elementos que conforman la definición o el diseño y la administración del currículo basado en una teoría curricular propia de cada institución educativa. Es sin duda, el aspecto sustancial de la gestión de la docencia y el que requiere un mayor grado de especialización y 
conocimientos técnicos. Gestión es un proceso y currículo es la representación de una realidad determinada, resultado de dicho proceso. Gestión curricular implica una respuesta no sólo a problemas de carácter educativo, sino también a los de carácter económico, político y social (p.31).

Los saberes necesarios para el estudio de la gestión curricular, demandan en primer lugar, abordar cuestiones curriculares, con la complejidad que supone definir "lo curricular". Desde esta perspectiva, se asume el currículum como una construcción cultural en dos sentidos: primero el currículum considera las circunstancias sociales e históricas que atraviesan la enseñanza institucionalizada y segundo el currículum también aparece atravesado, determinado en parte, contextualizado por la cultura institucional educativa propia de cada establecimiento, señala Poggi (1998, p.17).

Por lo tanto, la gestión curricular no se refiere sólo a simples procedimientos técnicos, sino que básicamente implica el proceso de dinamizar el desarrollo del currículo en sus diferentes fases, todas interdependientes entre sí. Además, la capacidad de organizar y poner en marcha el proyecto educativo y pedagógico de la institución con los elementos orientadores de la universidad. Asimismo, implica buscar un nivel de coherencia interna entre el pensamiento estratégico, los valores y los fines que propugna la universidad y los medios que utiliza para alcanzarlo como son los planes de estudio, los programas de asignaturas, las actitudes y la metodología docente. Así como la respuesta a las demandas y proyectos de formación de los pueblos.

\section{Modelo educativo centro de autonomía institucional y académica para una gestión curricular con identidad}

Los países latinoamericanos desde sus albores de vida republicana, diseñaron un tipo de educación que respondía a las necesidades educativas de sus clases dominantes que miraban más hacia afuera que hacia la realidad interior de los países a los que pertenecían. Por ello para muchos indígenas latinoamericanos, así como para muchos otros pueblos, la educación supone aún un forzado desaprendizaje de su propia cultura y un abandono, parcial si no total, de su lengua materna y su identidad. Esto significó para los pueblos, en muchos casos, asimilación, exclusión, invisibilización, negación, marginación, estereotipos, que han hecho mucho daño, hasta llegar a veces, a que los mismos pueblos nieguen su propia identidad.

Como bien explica Mato (2014) que, tanto los pueblos indígenas americanos, como los contingentes de personas africanas traídas a América en condiciones de esclavitud, padecieron varias circunstancias que atentaron especialmente contra importantes elementos constitutivos de sus visiones de mundo, uno de ellos es que sus espiritualidades fueron prohibidas y fueron forzados a adoptar el catolicismo, otro es que también sus lenguas fueron prohibidas, cuánto menos su uso en espacios públicos y especialmente en la escuela, cuando accedieron a ella (p.19). 
La historia de los sistemas educativos occidentales, leída desde la perspectiva intercultural, puede presentarse como la historia de una violencia, ya que promueve y posiciona la construcción de una identidad nacional homogénea e impuesta a toda la población en nombre de la modernidad, el progreso y la ciencia. Tal parece que los protagonistas de la construcción de los sistemas escolares hubiesen actuado impulsados por el lema "una nación, una lengua, una cultura" y en ocasiones "una religión" (Grupo Eleuterio Quintanilla, 1998).

A diferencia de las actitudes anteriores, la propuesta intercultural reconoce las diferencias, pero busca utilizarlas para potenciar encuentros fructíferos para generar niveles de unidad mayores, en los que el diálogo se de sobre la base del reconocimiento del otro a existir y a desarrollarse. No se pone aquí el énfasis en las barreras que deben servir a fortalecer internamente cada cultura, sino en los puentes que deben multiplicarse y ensancharse. Lo que reconoce una nueva atención a la diversidad cultural que reconoce la necesidad de promover, cada vez más, relaciones positivas entre los pueblos. Además de formar ciudadanos conscientes de las diferencias con capacidades de confrontar la discriminación, el racismo y toda forma de exclusión.

La asignación a la educación del papel de formar al ciudadano, nos hace que reflexionemos sobre la concepción ilustrada de ciudadanía hoy vigente entre nosotros, que se encuentra lamentablemente en extremo ligada de manera orgánica al antropocentrismo y al individualismo occidental moderno. De tal modo que nos parece natural que los derechos sean exclusivos de la especie a la que pertenecemos y que los derechos fundamentales sean por definición individuales, no colectivos.

La idea que no sólo los individuos sino también los colectivos son sujetos de derechos es difícil de asimilar a la concepción liberal clásica de los derechos. Sin embargo, los liberales multiculturalitas sí han ido más lejos y en ciertos casos es imprescindible formular derechos colectivos para hacer posible el ejercicio de los derechos individuales.

Así, por ejemplo, la educación, ha sido uno de las demandas centrales, en la lucha por los derechos de los pueblos indígenas, afrodescendientes y comunidades étnicas. La cual no debe ser vista únicamente como parte de la convivencia entre grupos culturalmente diferentes, sino como un tema estratégico, que permite en estos tiempos de globalización e integración entre países una mejor comprensión de las culturas, para una convivencia armónica entre las diferentes naciones del mundo.

El tema cobra especial relevancia en Centroamérica, tanto por las condiciones históricas de su diversidad cultural, como por el resurgimiento del "tema indígena" después de la Campaña Continental 500 años de Resistencia Indígena, Negra y Popular, reflejado en la Declaración de Xelajú en 1991, al definir entre las tareas futuras, "luchar para que en el programa educativo de cada país se inserte la enseñanza del contenido de nuestra verdadera historia, pluricultural y multilingüe". 
Está ampliamente documentado, que los pueblos indígenas y comunidades étnicas tienen idiomas, patrimonio, culturas y conocimientos ricos y diversos, adquiridos de sus relaciones entre sí y sus ecosistemas, que se reproducen de generación en generación y que se manifiestan a través de sus tradiciones, creencias, costumbres y ceremonias. Esos conocimientos, han sido incorporados a sus prácticas, para tener acceso a otros conocimientos, transmitirlos y compartirlo (ONU, 1999).

Tradicionalmente la Educación Superior en el continente se ha sustentado en las razones expuestas desde el pensamiento occidental universal, por obra y gracia de la colonización del conocimiento, fuera del ser y del estar, peor aún sin ni siquiera entender el "estar siendo" que resume la dinámica del pensamiento propio de los pueblos. El cual es una manera distinta de acercarse a la realidad, considerando al ser humano como una hebra del tejido vivo.

Por lo que desde hace muchos años, los pueblos han venido luchando por tener su propio modelo educativo, que haga visible su realidad, y que responda a las necesidades y sus expectativas. Una educación que haga evidente su historia, su cultura, sus lenguas, su manera de ver el mundo, los sistemas integrados de conocimientos holísticos, de saberes, enraizado en la fuerza viva de la espiritualidad que permite el enriquecimiento de valores éticos, humanistas, y que pueda reflejar la riqueza espiritual, como fuente inagotable de sabiduría y de equilibrio armónico del ser humano con su entorno.

No cabe duda que para superar el legado colonial, las universidades deben ser pertinentes con la diversidad cultural. Coincidiendo con lo planteado por Hooker (2004) que:

...un sistema sólido de educación es uno de los instrumentos claves que pueblos históricamente oprimidos y empobrecidos pueden usar para construir un futuro mejor". Los problemas fundamentales de sociedades multiétnicas compartiendo un territorio común nunca serán fáciles de solucionar y soluciones de calidad de estos problemas siempre exigirán de nosotros un auto sacrificio en gran medida, compromiso constante, abundante creatividad, una amplia variedad de inteligencia y unidad de propósito que suele ser extremadamente escasa. Autonomía no es para los débiles de corazón y espíritu, autonomía es verdaderamente un reto patriótico (p.5).

Los planteamientos centrales han girado en torno a la necesidad de redescubrir y valorar las formas propias de educación, y por otro lado, promover cambios en las formas de educar en las escuelas y otras instituciones, de forma que se alcancen resultados positivos en el marco del respeto a la diversidad cultural. 
Aspectos que no son visualizados ni incluidos en un programa de acción tanto en la Reforma de Córdoba de 1918 como en la ley de Autonomía de la Educación Superior de Nicaragua. Lo que resulta hoy un tema imperativo de abordar de manera responsable en las universidades que deben valorar la diversidad cultural y promover relaciones interculturales equitativas y mutuamente respetuosas, tanto en su seno como en la sociedad.

Es a partir de ellas que han luchado por reformas legales, incluso constitucionales, que tiendan a hacer posible la materialización de esa consigna, comenzando por lograr el reconocimiento del carácter pluriétnico de los respectivos Estados nacionales, así como la existencia de formas de ciudadanía culturalmente diferenciadas.

Pese a ese rezago histórico, tal vez haya motivos para ser optimistas. Podríamos decir que el contexto que vive América Latina, ha colocado como elemento central en los lineamientos de los organismos internacionales la elaboración de políticas públicas, a favor de la educación intercultural. No podemos desconocer el "avance" y promoción de normativas a favor de la revalorización y respeto por la cultura de los pueblos originarios y afrodescendientes, otrora (in) visibilizados y asimilados.

Al respecto, entre otras, viene al caso destacar que el artículo 26 del Convenio 169 de la OIT establece que: "deberán adoptarse medidas para garantizar a los miembros de los pueblos interesados la posibilidad de adquirir una "educación a todos los niveles", por lo menos en pie de igualdad con el resto de la comunidad nacional". En tanto, el artículo 27, entre otras cosas, dispone lo siguiente:

Los programas y los servicios de educación destinados a los pueblos interesados deberán desarrollarse y aplicarse en cooperación con estos, a fin de responder a sus necesidades particulares, y deberán abarcar su historia, sus conocimientos y técnicas, sus sistemas de valores y todas sus demás aspiraciones sociales, económicas y culturales, así como que la autoridad competente deberá asegurar la formación de miembros de estos pueblos y su participación en la formulación y ejecución de programas de educación, con miras a transferir progresivamente a dichos pueblos la responsabilidad de la realización de esos programas, cuando haya lugar; [finalmente estipula que] los gobiernos deberán reconocer el derecho de esos pueblos a crear sus propias instituciones y medios de educación, siempre que tales instituciones satisfagan las normas mínimas establecidas por la autoridad competente en consulta con esos pueblos. Deberán facilitárseles recursos apropiados con tal fin.

La Declaración de las Naciones Unidas sobre los derechos de los pueblos indígenas expresa, que la Asamblea General, preocupada por el hecho de que los pueblos indígenas han sufrido injusticias históricas como resultado, entre otras cosas, de la colonización y de haber sido desposeídos de sus tierras, territorios y recursos, lo que 
les ha impedido ejercer, en particular, su derecho al desarrollo de conformidad con sus propias necesidades e intereses, establece que:

los pueblos indígenas tienen derecho a todos los niveles y formas de educación del Estado. Todos los pueblos indígenas también tienen este derecho y el derecho a establecer y controlar sus sistemas e instituciones docentes impartiendo educación en sus propios idiomas y en consonancia con sus métodos culturales de enseñanza y aprendizaje (Art. 14).

También plantea que "los pueblos indígenas tienen derecho a que la dignidad y diversidad de sus culturas, historias, tradiciones y aspiraciones queden debidamente reflejadas en todas las formas de educación y en la información pública" (Art. 15).

La Conferencia Regional de la Educación Superior en América Latina y el Caribe (CRES, 2008) en la cual participaron más de 3.500 integrantes de la comunidad académica regional (incluyendo directivos, docentes, investigadores, estudiantes, funcionarios, representantes de organismos nacionales, regionales e internacionales y otros interesados en Educación Superior), reconoce la multiculturalidad de nuestros países, por lo que declara: "Nuestra región es marcadamente pluricultural y multilingüe. La integración regional y el abordaje de los desafíos que enfrentan nuestros pueblos requieren enfoques propios que valoren nuestra diversidad humana y natural como nuestra principal riqueza".

Además en su Declaración Final incluye algunos valiosos planteamientos sobre el tema. Así, en su acápite $C$-3 sostiene:

Se deben promover la diversidad cultural y la interculturalidad en condiciones equitativas y mutuamente respetuosas. El reto no es sólo incluir a indígenas, afrodescendientes y otras personas culturalmente diferenciadas en las instituciones tal cual existen en la actualidad, sino transformar a éstas para que sean más pertinentes con la diversidad cultural. Es necesario incorporar el diálogo de saberes y el reconocimiento de la diversidad de valores y modos de aprendizaje como elementos centrales de las políticas, planes y programas del sector.

Considerando, según la UNESCO (2002), que la diversidad cultural es nuestro patrimonio común y la mayor oportunidad para la humanidad. Es una promesa de renovación y de dinamismo, el motor mismo de la innovación y del desarrollo. Es una invitación al diálogo, al descubrimiento y a la cooperación. En un mundo tan diverso, la destrucción de las culturas es un crimen y la uniformidad un callejón sin salida: todo estriba en valorizar, en un mismo impulso, la diversidad que nos enriquece y los derechos humanos que nos reúnen. 
Preocupados y preocupadas por la pertinencia de la educación superior en la sociedad contemporánea, la Declaración en el acápite D-4 agrega: "La Educación Superior, en todos los ámbitos de su quehacer, debe reafirmar y fortalecer el carácter pluricultural, multiétnico y multilingüe de nuestros países y de nuestra región".

Actualmente las constituciones de la mayoría de los países latinoamericanos reconocen el carácter multicultural, multiétnico y plurilingüe y les conceden a los pueblos indígenas derechos de idioma, identidad y otros de carácter cultural. Hasta el presente este reconocimiento está consagrado en las constituciones de quince países latinoamericanos, estos son: Argentina, Bolivia, Brasil, Colombia, Costa Rica, Ecuador, El Salvador, Guatemala, Honduras, México, Nicaragua, Panamá, Paraguay, Perú y Venezuela.

La Constitución Política de Nicaragua (1987) define en 9 artículos el carácter multiétnico del país y reconoce el derecho a la diversidad lingüística y cultural. En los mismos artículos se destaca la oficialidad de las lenguas de la Costa Caribe de Nicaragua, la protección por parte del Estado del patrimonio lingüístico, el derecho de las y los costeños a preservar y desarrollar su identidad lingüística-cultural sin discriminación alguna, el reconocimiento del régimen autonómico, el acceso libre a la educación de los pueblos de la Costa Caribe, y la garantía por parte del Estado de la preservación de las culturas, lenguas, religiones, costumbres y medio ambiente.

Todo lo anterior demuestra que las demandas de reconocimiento y de educación planteadas por los pueblos indígenas, afrodescendientes y comunidades étnicas, han tenido eco en los diversos instrumentos jurídicos internacionales, nacionales y regionales. Además que el educativo es uno de los campos en los cuales pueden observarse algunos avances, aunque insuficientes, frecuentemente éstos resultan más significativos en el nivel normativo que en el de las realizaciones prácticas, ya que los Estados y gobiernos no se han organizado de manera efectiva para hacer realidad que la Educación Superior sea pertinente con la diversidad cultural de los países latinoamericanos (Mato, 2008a, p.21).

Como sostiene Falaschi (1998), la normativa existente más bien funciona como una declaración de principios "del deber ser", más que una real operatividad. Por lo que evidentemente no basta que tengamos un marco jurídico que reconozca los derechos colectivos de los pueblos, si no contamos con instrumentos que se logren operativizar en su ejercicio para hacer realidad sus postulados. Lo que (Mato, 2009), nos anuncia, como la «interculturalidad de hecho», para comprender mejor las características de los proyectos que apuntan a construir formas de «interculturalidad deseable», o «deseada».

Entendiendo la interculturalidad como el proceso permanente de construcción, establecimiento y fortalecimiento de espacios de diálogo, comunicación e interacción horizontal de doble vía, entre personas, comunidades y pueblos de diferentes culturas. 
La interculturalidad comprende el desarrollo de procesos de toma de decisiones conjuntas en igualdad de condiciones. Su finalidad es la de la promoción y práctica de la equidad, el respeto, la comprensión, la aceptación mutua y la creación de sinergias para el establecimiento de sociedades inclusivas, no discriminatorias y libres de racismo (URACCAN, 2014).

No olvidando que la interculturalidad como sistema de relaciones es complejo y debe fundamentarse en los principios de reciprocidad, voluntad, conocimiento, valoración, entendimiento, interacción, participación, horizontalidad, respeto y solidaridad entre las culturas. Siendo así, podemos considerar el sistema educativo en uno de los contextos más importantes para desarrollar y promover la interculturalidad, ya que es la base de la formación humana y un instrumento no sólo de mantenimiento de una sociedad, sino de desarrollo, crecimiento, transformación y liberación de esa sociedad y de todas sus potencialidades humanas.

El asunto clave es que se debe considerar a la educación como el espacio para reflexionar y repensar las diferentes formas de asumir la diversidad del ser humano, con el propósito de contribuir con el establecimiento de convivencias armónicas y construir sentidos de nación incluyentes. Esto supone proyectar la comprensión de las diversas prácticas socioculturales de las comunidades indígenas, afrodescendientes y mestizas, las cuales hemos venido reconociendo desde los múltiples proyectos individuales y colectivos que coexisten y configuran procesos de vida respectivos.

En lo particular, es importante destacar que varias de las nociones, conceptos y categorías más fundamentales que se han creado desde las ciencias actuales, violentan, desestructuran e imposibilitan la auto comprensión desde la pertinencia indígena y afrodescendiente. Siendo necesario entonces, buscar la forma de abrir espacios interculturales que den cabida hacia nuevas reflexiones, debates y discusiones que contribuyan a crear una nueva condición social del saber.

Según Walsh (200o), el problema dentro del sistema educativo, es cómo tratar la diversidad cultural, asunto que hasta el momento, no ha recibido una atención amplia, crítica y concreta sobre cómo profundizar la unidad de lo diverso, cómo promover la interculturalidad y cómo formar ciudadanos capaces de practicar la equidad y de confrontar las tensiones que caracterizan el siglo XXI: la tensión entre lo mundial y lo local; la tensión entre lo universal y lo propio; la tensión entre tradición y modernidad.

Incluir la interculturalidad como elemento básico del sistema educativo implica que se asume la diversidad cultural desde una perspectiva de respeto y equidad social, una perspectiva que todos los sectores de la sociedad tienen que asumir hacia los otros. Este aspecto tiene que partir de la premisa de que todas las culturas tienen el derecho a desarrollarse y a contribuir, desde sus particularidades y diferencias, a la construcción del país. Eso quiere decir, como expresa Haro y Vélez (1997): 
Que no se jerarquicen determinados tipos de conocimientos sobre otros - saber formal/saber tradicional-y que no sean solamente los actores débiles de la sociedad quienes estén en la obligación de conocer la cultura de los actores dominantes: sus lenguas, sus costumbres, sus códigos de comportamiento, sino que más bien la educación se dé en términos de igualdad de condiciones, sin discriminación e irrespeto... Para que la educación sea realmente intercultural, es preciso que todas las culturas implicadas en el proceso educativo se den a conocer y se difundan en términos equitativos: con maestros indios, afros, hispano-hablantes y extranjeros; contemplando en los contenidos curriculares los múltiples elementos de conocimiento y sabiduría de las diferentes culturas; factibilizando la enseñanza con metodologías diversas y adecuadas a la realidad pluricultural; con recursos didácticos funcionales...Si la pretendida educación intercultural no toma en cuenta desde la praxis la diversidad cultural del país, será un intento parecido a muchos otros, cuyo único resultado ha sido la asimilación de la cultura de las minorías étnicas a la cultura nacional y dominante (p. 302-303).

Por tanto, la interculturalidad debe ayudarnos a construir relaciones equitativas y dialógicas con personas de distintas tradiciones socioculturales, quizá éste sea el nivel más importante y difícil de trabajar. Conocer y valorar a los "otros", pero, especialmente, desarrollar una actitud de reconocimiento y respeto a sus derechos es un reto para todos y todas. Frente a estas premisas la respuesta del modelo convencional de universidad resulta insuficiente. La situación es aún menos favorable según plantea Mato (2009), si nos preguntamos por la existencia de instituciones o programas que respondan de manera explícita a necesidades e intereses expresados por comunidades y pueblos indígenas y afrodescendientes, que incorporen los saberes de estos pueblos, sus lenguas y modalidades de aprendizaje en los planes de estudio, o que contribuyan deliberadamente a la valoración y promoción de la diversidad cultural y de relaciones interculturales equitativas y de valoración mutua.

Aunque podemos resaltar la disponibilidad y disposición política de las Universidades convencionales a atender la diversidad cultural, poniendo en práctica algunas acciones afirmativas hacia los pueblos. Entre las que se pueden citar, la apertura de cupos especiales y programas de becas a estudiantes indígenas y afrodescendientes que mejoran las posibilidades de acceso y culminación de estudios de Educación Superior en Instituciones de Educación Superior "convencionales".

Lo que no es suficiente dentro de su vieja institucionalidad que es expresión viva del legado colonial. Adicionalmente, debe señalarse que esas políticas y programas están orientadas a lograr "la inclusión de individuos", por lo que, aunque desde ciertos puntos de vista constituyen un avance, no obstante no resuelven el problema de que los programas de estudio y líneas de investigación de las universidades en cuestión, 
continúan excluyendo tanto las historias, lenguas, valores, conocimientos, prácticas y saberes de esos pueblos. Así como sus necesidades, demandas, propuestas y proyectos sociales, políticos y económicos, para sí mismos y para las sociedades nacionales de las que forman parte.

Por lo mismo, estas políticas y programas tampoco resultan suficientes para formar los cuadros técnicos, profesionales, gerenciales y políticos, que esos grupos de población, sus organizaciones sociales, y los horizontes de transformación demandan, plasmados en las nuevas constituciones nacionales.

Es importante destacar, que desde la propuesta de educación intercultural, se parte del reconocimiento de los derechos de los pueblos indígenas, afrodescendientes y comunidades étnicas desde dos vertientes: el derecho a la educación como todos los demás ciudadanos/ciudadanas, y el derecho a la revitalización y desarrollo de su propia cultura. Además responde a la necesidad de contar con sistemas educativos pertinentes y de calidad, base fundamental para el desarrollo sostenible. Garantizar lo anterior, requiere de un abordaje de la equidad que demanda como primer paso, compartir valores y principios entre toda la sociedad, el Estado y los pueblos indígenas.

La Educación Superior Intercultural, además de ser un espacio de fortalecimiento de los conocimientos, nos permite lograr una apertura democrática y adquirir las herramientas para incidir en las reformas políticas que deben realizar los estados a nivel comunal, territorial, municipal, regional e internacional.

Actualmente podemos observar algunos avances significativos en el campo de la Educación Superior Intercultural, pero todavía siguen siendo insuficientes para la verdadera «interculturalización» de la Educación Superior, entendida como la inclusión de los conocimientos y modos de producción de conocimientos, formas de aprendizaje, lenguas, historias, visiones de mundo, sobre todo, la conservación y pervivencia como pueblos indígenas y afrodescendientes, en los planes de estudio de todas las IES, según sea pertinente para las diversas formaciones profesionales.

En ejemplo, podemos citar el Plan Estratégico de la Educación Superior de Nicaragua 2012-2021 (CNU, 2012), el cual plantea la Interculturalidad como un eje transversal y uno de los principios rectores de la Educación Superior nicaragüense. Donde se plantea que es un proceso de relación, negociación e intercambio cultural, que conduce al desarrollo de una interacción entre personas y colectividades, conocimientos, saberes y prácticas culturalmente diferentes, fomentando la igualdad como un elemento de identidad y oportunidades para el desarrollo. Por otro lado, considera la diversidad étnica, lingüística, cultural, técnica, científica y humanista, al reconocer los diferentes sistemas educativos y enfoques que se implementan en la creación y recreación de conocimientos, saberes y prácticas de los pueblos. Incorpora el diálogo de saberes y el reconocimiento de la diversidad de valores y modos de aprendizaje 
como elementos centrales de las políticas, planes y programas educativos, como respuesta a las demandas de atención a la diversidad cultural y en correspondencia con la pertinencia social.

La tarea de "interculturalizar toda la educación superior", de hacerla verdaderamente "universalista", y no monocultural y subalternamente seguidora del legado europeo moderno, y de igual forma articulada al mercado mundial, sigue pendiente (Mato 2008b, 2008c). Son muy pocas las Instituciones de Educación Superior de América Latina que incorporan los conocimientos, lenguas, propuestas, y modalidades de aprendizaje de esos pueblos en sus planes de estudio, y que contribuyen deliberadamente a la valoración de la diversidad cultural, la promoción de relaciones interculturales equitativas y de formas de ciudadanía que aseguren la igualdad de oportunidades.

La Reforma de Córdoba de 1918 se propuso redistribuir el poder o el gobierno de la Universidad para forzar su apertura a las clases medias emergentes; los procesos de reforma académica de los años 50 y 60 enfatizaron aspectos como la educación general, la departamentalización, la reinstalación de la ciencia y la investigación en el quehacer de la Universidad para superar el sobreénfasis profesionalista heredado del modelo napoleónico. En la década de los 90 se habla más bien de procesos de transformación universitaria, encaminada a mejorar la calidad, la pertinencia, la gestión, el diseño curricular, y los métodos de enseñanza-aprendizaje (Tünnermann, 20011, p. 10).

Será entonces la gestión curricular intercultural, como componente medular de la acción educativa, el medio con el cual esperamos fortalecer el conocimiento, conservación y manejo de los recursos naturales "el cuidado a nuestra madre tierra", esperamos fortalecer la dirigencia indígena y las formas propias de gobierno, defender nuestros derechos, gestionar a nivel nacional e internacional proyectos en materia educativa, fortalecer la enseñanza en materia de producción, para el caso específico de URACCAN, fortalecer nuestra propuesta en materia de educación y salud.

Además de lo anterior, debemos darle respuesta con el accionar a algunas interrogantes como: ¿La universidad contribuye a través de sus currículos a fortalecer la identidad cultural de los y las estudiantes indígenas y afrodescendientes? ¿Los egresados de las universidades son profesionales al servicio de sus comunidades y de los procesos político-organizativos de sus pueblos? ¿Cuál es el efecto de la presencia de estudiantes indígenas y afrodescendientes en el ambiente universitario? ¿Contribuye dicha presencia a la apertura y enriquecimiento cultural de la universidad? ¿Cómo enfocan las universidades el tema de la interculturalidad? ¿Qué condiciones se requieren para los diálogos de saberes? ¿La diversidad cultural y por tanto la educación intercultural, se reduce a lo étnico? ¿Se trata de crear programas especiales para indígenas y afrodescendientes, o de construir una sociedad incluyente de la diversidad? 
De ahí la necesidad de reflexionar sobre el quehacer de las Universidades frente a la realidad de una diversidad cultural potencial y el ejercicio efectivo de la autonomía docente o académica. Considerando lo planteado por Hooker (2008):

las universidades deben contribuir a que los pueblos originarios y comunidades afrodescendientes puedan ejercer sus derechos a practicar y revitalizar sus tradiciones y costumbres culturales, lo que implica el derecho a revitalizar, utilizar, desarrollar y transmitir a las generaciones futuras sus historias, idiomas, tradiciones orales, filosofías, sistemas de escritura y literaturas, debiendo el Estado proporcionar los medios necesarios para tal cometido (p.9).

Es así que sólo mediante el pleno ejercicio de una verdadera autonomía universitaria y una plena libertad académica es que las universidades pueden apropiadamente, desde sus propios proyectos educativos, currículos y de la misión y visión que de ellos se desprende, promover su pertinencia y atención a la diversidad cultural frente a las demandas, sueños, aspiraciones y proyectos de los pueblos. En este sentido, la autonomía, asumida responsablemente y como una autonomía dinámica y de presencia en la sociedad, lejos de ser un obstáculo a la relevancia de las instituciones de educación terciaria, es la mejor garantía para que el quehacer de la educación superior responda a las demandas y desafíos del entorno nacional, regional e internacional.

Si tomamos como referencia lo planteado por Tünnermann (1990) que: "la Autonomía Universitaria es de la esencia misma de la Universidad, ya que sin ella se desfigura, cuando no se destruye, la función propia de la Universidad: ser órgano de ciencia y útil instrumento de orientación en una colectividad" (p.9).

Entonces algunos de los aspectos que no pueden quedar excluidos son los siguientes: en primer término, las universidades tienen que ser pertinentes con el contexto social y responder de una manera coherente con la diversidad cultural. Donde el proyecto educativo deje explicito qué (qué se enseña) y el cómo (cómo se enseña), que conduce a analizar los contenidos curriculares y los métodos de enseñanza-aprendizaje.

Entonces, un desafío pendiente lo constituye la puesta en marcha del currículo destinado a ésta educación, ¿quiénes participan del mismo y con qué peso en las decisiones curriculares? ¿Y cuánto del mismo sigue aún siendo de corte monoculturaleurocentrado, asociado al trabajo individual, no cooperativo, en aulas, bibliotecas y laboratorios?

En efecto, no se trata de reproducir una educación superior que replique el sistema de conocimiento vigente, donde los conocimientos de los pueblos, en el mejor de los casos, son un aspecto circunstancial en el currículo académico. Tampoco se pretende crear un espacio de saber, reservado solamente para los indígenas y afrodescendientes, 
sino de construir contenidos fundamentales que permitan vivenciar la interculturalidad, potenciando la riqueza de los saberes ancestrales, emprender un diálogo integral que efectivamente supera la ruptura entre lo teórico y lo práctico desde lo intercultural, lo que requiere la construcción de nuevos marcos conceptuales, analíticos, en los cuales se vayan generando nuevas formas de pensar, sentir y actuar, bajo el marco de la interculturalidad.

Debe permitir el aprendizaje producto del diálogo de saberes locales y occidentales, mirando críticamente ambos, pero con mayor alerta al dominante y hegemónico. Para que la propuesta de educación intercultural, no quede bajo sospecha permanente, vinculada sólo a la educación compensatoria, destinada a ocultar la desigualdad y a mantener la gobernabilidad de los Estados.

Trabajar la interculturalidad en la universidad, implica dar una mirada distinta a una serie de aspectos de nuestra labor educativa. Exige repensar toda nuestra práctica docente y analizar su pertinencia a la luz de las características socioculturales de los estudiantes con quienes trabajamos y de sus necesidades como personas y como miembros de un pueblo. Nos desafía a revisar las competencias que queremos desarrollar, los contenidos que vamos a trabajar, las estrategias que vamos a usar y los criterios y procedimientos con los cuales vamos a evaluar.

La estructura curricular bajo el criterio de interculturalidad incorpora los saberes, conocimientos, prácticas, valores y cosmovisiones de los pueblos, así como la ciencia y la tecnología moderna, contribuyendo a este modelo un proceso lógico, coherente e integral en todos sus niveles, favoreciendo preservar, rescatar y promover las culturas.

De ahí que la gestión curricular debe promover y llevar a cabo principios metodológicos que orientan esfuerzos hacia la construcción y reconstrucción de conocimientos apoyados en los conocimientos, prácticas y saberes endógenos. Mediante la investigación, crear y recrear conocimientos, saberes y vincular las prácticas de las aulas con la realidad objetiva del entorno a través de un proceso participativo, autogestionario y dinámico de los ciudadanos y ciudadanas interculturales que propicia su empoderamiento y a la vez genera capacidades para transformar las sociedades a las que pertenecen. Reconocer y asumir, que los pueblos son portadores milenarios de conocimiento y saberes, que sus culturas enriquecen la cultura global, que son fuentes teóricas y prácticas científicas para el autodesarrollo en el marco de una nueva relación horizontal con el mundo. Asimismo, presentar la sabiduría como una proposición favorable para comprender el saber y el conocimiento desde los pueblos, que no asume la implicación de la razón como herramienta suprema para determinar la valía de un conocimiento que piensa en la vida.

Además, mediante la investigación y el acompañamiento comunitario se debe llevar a cabo la recuperación, la comprensión y la utilización de los conocimientos propios 
de las comunidades, incluyendo conocimientos sobre la naturaleza, la vida social, la territorialidad, y sobre la ciencia y la matemática. Los cuales son pasos esenciales para fortalecer la identidad cultural propia y construir relaciones (interculturales) de conocimiento menos asimétricas, entre pueblos y con el mundo occidental.

Los centros productivos de las comunidades le debe permite a la Universidad tener un laboratorio invaluable para actividades prácticas y de investigación, ya que contribuyen a la formación científica del estudiantado y también lo prepara como ser humano que comparte, valora y respeta los conocimientos, prácticas y cosmovisiones de los pueblos. Por lo que las metodologías deben permitir a las universidades salir de sus entornos y adentrarse a los espacios donde se hace la vida, se hace el conocimiento, trabajar con los sujetos de estos procesos, validarlos y divulgarlos para que obtengan el reconocimiento que por tanto tiempo le ha sido negado.

Pero no podemos perder de vista que el verdadero quid de la transformación reside en los métodos pedagógicos y en la práctica docente, donde la gestión curricular juega uno de los papeles más importantes en la realización del proyecto educativo. Es así que la gestión curricular intercultural de la Universidad debe centrase en la construcción de la interculturalidad, pensada y expresada en los planes de estudio y estrategias de aprendizaje como algo no separado entre diseño y desarrollo, sino que se une en la práctica cotidiana en las aulas a partir de las concepciones de los pueblos indígenas y/o afrodescendientes, de sus visiones de mundo, de sus interpretaciones de la historia humana, de sus aprendizajes y de sus propuestas de futuro.

Donde se promueva y profundice en una educación de carácter humanista, la cual debe estar orientada a la formación integral de personas, ciudadanos y profesionales competentes, que tengan impregnado el respeto y defensa de los derechos humanos, la lucha por la igualdad y la justicia social, el combate contra toda forma de discriminación, el diálogo intercultural con pleno respeto a las identidades y un fuerte espíritu de la unidad en la diversidad.

\section{Universidades indígenas, interculturales y comunitarias}

Es importante resaltar que aunque en número escaso existen experiencias que han ido surgiendo y ganando lugar como instituciones con perfiles particulares que incorporan la interculturalidad como enfoque, principio, valor y metodología en su quehacer, como las Universidades Indígenas, Interculturales y Comunitarias, que valoran la diversidad humana y natural como la principal riqueza.

Las mismas son parte de las naciones originarias, pueblos y comunidades indígenas y afrodescendientes articuladas y vinculados a los movimientos indígenas y sociales de cada país. Se considera que son el espacio de recuperación, fortalecimiento y acompañamiento de los planes y propuestas de vida de los pueblos y sus organizaciones 
para impulsar el Buen Vivir, el Vivir Bien y el Vivir con Dignidad. Donde se vincula de manera efectiva la teoría y la práctica en la perpetuación de los procesos de aprendizajes, creación y recreación comunitaria de sistemas de conocimiento indígenas, fundamentados desde el conocimiento ancestral. Se implementan metodologías que facilitan el diálogo e interacción de los diferentes saberes en un marco de construcción colectiva de conocimientos. Además se recupera y revitaliza el idioma de las naciones y pueblos indígenas y afrodescendientes de Abya Yala, y se desarrolla la espiritualidad propia. En general, en estas universidades se busca la armonía entre la razón y el corazón para la solución de los problemas de formación, en tanto se fundamente en el sentir, pensar y actuar en un contexto de diversidad.

Donde los principales logros que se pueden destacar hasta el momento que son: 1). Mejoran las posibilidades de que individuos indígenas y afrodescendientes accedan a oportunidades de Educación Superior y culminen exitosamente sus estudios; 2). Ajustan su oferta educativa a necesidades, demandas y proyectos de las comunidades y la relacionan con oportunidades locales y regionales (subnacionales) de empleo, generación de iniciativas productivas y servicio a la comunidad; 3 ). Desarrollan modalidades participativas de aprendizaje, frecuentemente centradas en la investigación aplicada; 4). Integran aprendizaje, investigación y servicio a las comunidades; 5). Integran diversos tipos de saberes y modos de producción de conocimiento; 6). Promueven la valorización e incorporan las lenguas y saberes propios de estos pueblos y comunidades, contribuyen proactivamente a su fortalecimiento y realizan investigación sobre dichas lenguas y saberes; 7). Desarrollan docencia e investigación orientadas por criterios de valoración de la diversidad cultural, interculturalidad, equidad, inclusión, gobernabilidad democrática, desarrollo humano y sostenible; 8). Forman egresados que contribuyen al desarrollo sostenible local y regional, y al mejoramiento de la calidad de vida de sus comunidades (Mato, 2008a, p. 19).

Pero quedan muchos desafíos y retos por los cuales trabajar en una Educación Superior Intercultural con la calidad que queremos y pertinencia respecto de la diversidad cultural propia de cada uno de los pueblos, que contribuya a fortalecer las identidades de cada pueblo y a potenciar las condiciones para lograr un buen vivir comunitario centrado en la unidad, diálogo, reciprocidad o correspondencia; también en el fortalecimiento y construcción de autonomía, así como la capacidad para proyectarse y articularse a otras sociedades respetando los derechos de todos y todas.

\section{Conclusiones}

El ejercicio de una verdadera Autonomía implica el fortalecimiento de nuestros valores culturales y de nuestra identidad como naciones. Abiertas al diálogo intercultural y a la comunidad académica que vigoriza proyectos educativos pertinentes a la diversidad cultural en contextos multiculturales. 
Es de vital importancia reconocer el carácter colonial de la educación superior que ha marcado a los pueblos para poder llevar a cabo una efectiva y pertinente reforma curricular. Así como reflexionar que todo cambio curricular debe ir acompañado de un cambio estructural institucional, considerando la gestión como componente esencial para el cumplimiento del proyecto educativo institucional.

Se debe trabajar en la interculturalización de toda la educación superior como un cumplido y una deuda pendiente a la pertinencia social y calidad de toda universidad en contextos multiculturales.

\section{Lista de referencias}

Álvarez, B. Ma. G. (1998). "La administración de la curricularización. Desarrollo de la actitud política de la institución educativa universitaria”, en Gestión docente universitaria. Modelos comparados, vol. 2, Santiago de Chile, Centro Interuniversitario de Desarrollo cinda, pp. 47-6o.

CINDA y ALFA (1996). Gestión docente universitaria: modelos comparados. Volumen No. 2. Santiago, Chile.

Constitución Política de la República de Nicaragua (2007). Managua, Nicaragua.

CRES (2008). Declaración y Plan de acción de la Conferencia Regional de Educación Superior en América Latina y el Caribe. Organización de las Naciones Unidas para la Educación la Ciencia y la Cultura. Instituto Internacional de la UNESCO para la Educación Superior en América Latina y el Caribe.

FALASCHI, C: (1998). El discurso de la educación bilingüe e intercultural en Argentina. In: Congreso chileno de antropología, 3, Temuco [Anais...] Temuco: Universidad Católica de Temuco.

Grupo Eleuterio Quintanilla (1998). Libros de texto y diversidad cultural. Talasa, Madrid.

Haro, H. y Vélez, C. (1997). "La interculturalidad en la reforma curricular", De la protesta a la propuesta. Memorias de los talleres de antropología aplicada. Quito, Universidad Politécnica Salesiana.

Hooker, A. (2008). Educación superior y ciencia y tecnología en América Latina y el Caribe: Respuestas frente a la expansión y a la diversificación. Ponencia I Congreso Nacional de Educación Superior en Nicaragua. 
Hooker, R. (2004). Discurso inaugura del IV Simposio de Autonomía "Para la reflexión de los aportes a la construcción de una nación intercultural”, Managua, Nicaragua: URACCAN.

Mato, D. (2008a). Diversidad cultural e interculturalidad en Educación Superior. Experiencias en América Latina, IESALC-UNESCO. Recuperado de http:// www.iesalc.unesco.org.ve

Mato, D. (2008b). Actualizar los postulados de la Reforma Universitaria de 1918: las universidades deben valorar la diversidad cultural y promover relaciones interculturales equitativas y mutuamente respetuosas. En: Emir Sader, Pablo Gentili y Hugo Aboites (compiladores) La reforma universitaria: desafíos y perspectivas noventa años después. Buenos Aires: Consejo Latinoamericano de Ciencias Sociales (CLACSO): pp. 136-145.

Mato, D. (2008c). No hay saber "universal", la colaboración intercultural es imprescindible. Alteridades 18 (35): pp. 101-116.

Mato, D. (2009). Educación superior, colaboración intercultural y desarrollo sostenible / buen vivir. Experiencias en américa latina.

Mato, D. (2014). Universidades Indígenas en América Latina. Experiencias, logros, problemas, conflictos y desafíos. Revista ISEES, No 14, julio - diciembre 2014, pp.17-45.

Naciones Unidas (2007). Declaración de las Naciones Unidas sobre los derechos de los pueblos indígenas. 107a. sesión plenaria 13 de septiembre de 2007.

Organización Internacional del Trabajo. Oficina Regional para América Latina y el Caribe. Lima, Perú (2007). Convenio No. 160 sobre Pueblos Indígenas y Tribales en países independientes.

ONU (1999). Informe sobre el seminario sobre la educación superior y los pueblos indígenas. Comisión de Derechos Humanos.

Orozco, L. E. (1994). Conferencia en evento sobre Ciencia, Tecnología y Desarrollo Humano. Universidad Tecnológica de Pereira.

CNU (2012). Plan Estratégico de la Educación Superior en Nicaragua 2012-2021. Consejo Nacional de Universidades de Nicaragua.

Poggi, M (comp.) (1998). Apuntes y aportes para la gestión curricular. Colección triángulos pedagógicos. Buenos Aires, Argentina: Kapelusz. 
Tünnerman, C. (1996). La Educación Superior en el umbral del Siglo XXI. UNESCO CRESALC, Caracas.

Tünnermann, C. (1990). Breve Reseña de la Conquista de la Autonomía Universitaria en Nicaragua. Recuperado de http://www. enrique bolaños.org.

Tünnermann, C. (1997). Aproximación histórica a la universidad y su problemática actual. Primera Edición, Santafé Bogotá, Colombia: Universidad de los Andes.

Tünnermann, C. (2011). Pertinencia y calidad de la educación superior. Seminario "Políticas educativas de evaluación de la educación superior en América Latina y el Caribe desde el contexto de la pertinencia". Universidad del Atlántico, Barranquilla, Colombia. Recuperado de http://desarrollopedagogico.files.word.

UDUAL (1954). Acuerdos del Segundo Congreso Universitario y Primera Asamblea General de la Unión de Universidades Latinoamericanas. Santiago de Chile: Universitaria.

UNESCO (2002). Declaración universal de la UNESCO sobre la diversidad cultural. Adoptada por la 31a reunión de la Conferencia General de la UNESCO, PARÍS.

URACCAN (2014). Planificación Estratégica 2015-2019.

Walsh, C. (2000). Propuesta para el tratamiento de la Interculturalidad en la Educación. Lima Perú. 